\title{
LETTER
}

\section{A "metaphorical" nonlinear multimode fiber laser approach to weakly dissipative Bose-Einstein condensates ${ }^{a}$}

To cite this article: V. L. Kalashnikov and S. Wabnitz 2021 EPL 13334002

View the article online for updates and enhancements. 


\title{
Focus Article
}

\section{A “metaphorical” nonlinear multimode fiber laser approach to weakly dissipative Bose-Einstein condensates ${ }^{(a)}$}

\author{
V. L. KalashniKOV ${ }^{1,2}$ and S. WABnitz ${ }^{1,3}$ \\ 1 Dipartimento di Ingegneria dell'Informazione, Elettronica e Telecomunicazioni, Sapienza Università di Roma \\ via Eudossiana 18, 00184 Rome, Italy \\ ${ }^{2}$ Institute of Photonics, Vienna University of Technology - Gusshausstrasse 25-29, Vienna 1040, Austria \\ 3 Novosibirsk State University - Pirogova 1, Novosibirsk 630090, Russia
}

received 15 October 2020; accepted in final form 11 February 2021

published online 26 March 2021

PACS 42.65.-k - Nonlinear optics

PACS 03.75.Lm - Tunneling, Josephson effect, Bose-Einstein condensates in periodic potentials, solitons, vortices, and topological excitations

PACS 42.81.Dp - Propagation, scattering, and losses; solitons

\begin{abstract}
We demonstrate the stabilization of two-dimensional nonlinear wave patterns by means of a dissipative confinement potential. Our analytical and numerical analysis, based on the generalized dissipative Gross-Pitaevskii equation, makes use of the close analogy between the dynamics of a Bose-Einstein condensate and that of mode-locked fiber laser, operating in the anomalous dispersion regime. In the last case, the formation of stable two-dimensional patterns corresponds to spatiotemporal mode locking, using dissipation-enhanced mode cleaning. We analyze the main scenarios of pattern destabilization, varying from soliton dissolution to its splitting and spatiotemporal turbulence, and their dependence on graded dissipation.
\end{abstract}

Introduction. - Emerging phenomena comprising turbulence, light, matter and quasi-particle Bose-Einstein condensation, including the transition between coherent and non-coherent collective states mediated by spatiotemporal turbulence [1-8], bridge macro- and quantum physics, and open the way to a "mesoscopic" quantum world [9]. An impressive example of coherent quantum mesoscopic state is provided by Bose-Einstein condensation (BEC). BEC can be treated as a matterwave soliton, similar to the soliton solution of the nonlinear Schrödinger equation (NSE) in optics [10,11]. Such analogy is based on the equivalence of the underlying onedimensional (1D) models, where time coordinate, kinetic energy, and attractive interaction of bosons for a BEC correspond to propagation distance, anomalous groupdispersion (or diffraction for spatial solitons [12]), and selfphase modulation (self-focusing) in optics, respectively.

On the one hand, it is well-known that $2 \mathrm{D}$ solitons undergo a catastrophic collapse $[12,13]$. On the other hand,

(a) Contribution to the Focus Issue Turbulent Regimes in BoseEinstein Condensates edited by Alessandra Lanotte, Iacopo Carusotto and Alberto Bramati. it is also known that BEC solitons can be stabilized by means of introducing a trapping potential [14-17], or by tuning the effective inter-atom interaction potential from repulsive to attractive through a Feshbach resonance [18]. In particular, this can be achieved with a periodic temporal modulation of nonlinearity [19-23], or by means of a modulation of the trapping potential [23]. A whole zoo of different well-localized spatial coherent and semi-coherent states with non-zero topological charges was theoretically demonstrated (see [15] for an overview).

A similar stabilization methodology for ultra-short optical pulse propagation is essential in optical telecommunications, where the confinement potential can be introduced by means of a graded refractive index (GRIN) in optical fibers $[17,24]$. Moreover, the possibility of a periodical modulation of the group-velocity dispersion (GVD) in optical fiber systems, and the use of different nonlinearity mechanisms, may provide additional tools for spatiotemporal soliton stabilization $[23,25,26]$. The close analogy between nonlinear phenomena in atomic and photonic systems makes the latter an ideal test-bed for exploring fundamental physics, ranging from plasma to BEC [27]. 
An additional advantage of the analogy between BEC and nonlinear optical systems concerns the scaling of the boson amount, which is the BEC mass or the optical soliton energy, respectively. This issue has two principal aspects. The first one is the contribution of dissipative effects to the properties of BEC [28-30] and optical solitons [31]. Such a contribution can be crucial in the process of coherent condensate (or soliton) self-emergence, and its stability [26]. As was demonstrated, pulse manipulation with the help of a graded dissipation in a fiber laser could provide the means to achieve the self-emergence of stable dissipative solitons, by the so-called distributed Kerr lens mode-locking technique [32]. Our first proposal is to expand this method to BEC.

The second aspect concerns the scaling of the condensate mass/energy, which can be provided by varying the condensate size. In terms of fiber optics, this means using GRIN multimode fibers (MMFs), where spatial instabilities could destabilize pulse dynamics. It was found that the effect of nonlinear spatial mode cleaning in MMFs can suppress such instabilities, and provide a way to achieve spatiotemporal mode locking, i.e., the formation of localized stable coherent spatial and spatiotemporal patterns $[17,33,34]$.

In this letter, we consider a generalized dissipative model based on the 2D Gross-Pitaevskii equation (GPE), taking into account the presence of graded dissipation, and mass/energy exchange with a non-coherent environment. The analytical soliton solution corresponding to the ground soliton state (i.e., fundamental mode soliton in photonics) is demonstrated. Furthermore, the stabilization of $2 \mathrm{D}$ coherent structures by graded dissipation (dissipative mode cleaning) is numerically analyzed.

2D dissipative soliton. - Let us consider a particular case of BEC, formed by an axisymmetric harmonic potential of cigar type with confinement along $r$-axis, and unconfined along the $z$-axis. In photonics, this is a model for ultrashort pulse dynamics in a GRIN fiber laser (the $z$-coordinate corresponds to a local or "retarded" time coordinate in that case). The last statement is akin to a specific space-time duality in optical signal processing $[35,36]$. Thus, anomalous GVD plays the role of the $z$-component of the boson kinetic energy. The corresponding dimensionless master equation is the generalized 2D GPE:

$$
\begin{aligned}
i \frac{\partial \psi}{\partial T}= & -\frac{1}{2}\left[\frac{1}{r} \frac{\partial}{\partial r} r \frac{\partial}{\partial r}+(1-i \tau) \frac{\partial^{2}}{\partial z^{2}}\right] \psi+\frac{1}{2}(1-2 i \kappa) r^{2} \psi \\
& -|\psi|^{2} \psi-i\left(\Lambda+\sigma \int_{-\infty}^{\infty}|\psi|^{2} \mathrm{~d} z\right) \psi
\end{aligned}
$$

Here, axial symmetry and zero vorticity are assumed for the wave function $\psi(T, r, z)$ (which is a local-timedependent field amplitude in photonics). The first term defines the kinetic energy (diffraction/dispersion in photonics) with a "kinetic cooling" ("evaporation") along the unconfined $z$-axis. The last results from the growth of escaping rate with kinetic energy (that is, spectral dissipation in photonics) and it is defined by the $\tau$-parameter. The second term describes the complex (i.e., dissipative) parabolic confining potential, whose imaginary part $\kappa$ contributes because of the resonant interaction with the light field, or the dissipative nature of the confining lattice itself $[30,37]$. In photonics, this term characterizes a GRIN fiber profile, with a graded dissipation tracing the parabolic refractive index profile [32]. The parameters for a real-world fiber laser system correspond to table 1 in [32] and the caption to fig. 5 (see refs. $[57,58]$ in [32]). The case of dissipative BEC as discussed in [28] suggests the dimensionless values of $\left|\Lambda_{s}\right| \sim 0.001-0.1$, which coheres with the fiber laser parameters that we considered in this work. The third nonlinear term is related to the two-bodyscattering length. We consider an attractive interaction in a condensate, which defines the sign before this term: this corresponds to self-phase modulation (self-focusing) in photonics. The last term describes nonlinear loss caused by a weak dissipative condensate-basin interaction. Here, $\Lambda<0$ corresponds to a net linear gain (a "gain" means an inflow from non-coherent "reservoir" to the condensate) [30]. We impose a possible saturation of such gain with the condensate mass growth, as defined by the $\sigma$ parameter. This effect is an analog of the gain saturation in a laser. It should be pointed out that, for the case of $\mathrm{BEC}$, introducing a nonzero $\sigma$-term is a quite unusual procedure, which can be associated with the "exhausting" of an incoherent reservoir, interacting with the condensate. Nevertheless, as we are going to see in the following, the results presented in this work remain self-consistent also in the $\sigma \rightarrow 0$ limit.

The dissipative potential can be characterized by an effective width (or "aperture size") parameter $\chi=\sqrt{\left|\Lambda_{s}\right| / \kappa}$, where $\Lambda_{s} \equiv \Lambda+\sigma \int|\psi|^{2} \mathrm{~d} z<0$ is a net gain in eq. (1). This parameter describes dissipation-enhanced mode cleaning: for a fiber system, its physical meaning is associated with the spatial domain's size, outside of which loss effect begins to prevail [32]. The relevant dimensionful parameters are presented in the captions of figs. 5-7.

The approximate solution of eq. (1) corresponding to the soliton-like ansatz

$$
\begin{aligned}
& \psi(T, r, z)=\psi_{0}(T) \operatorname{sech}\left(\frac{z}{\mathrm{Z}(T)}\right) \\
& \times \exp \left[i\left(\phi(T)+\zeta(T) z^{2}+\theta(z) r^{2}\right)-\frac{r^{2}}{2 \mathrm{P}(T)^{2}}\right],
\end{aligned}
$$

where $\psi_{0}, \mathrm{P}, \mathrm{Z}, \zeta, \theta$, and $\phi$ are the time-dependent amplitude, spatial widths, chirp, and phase, respectively, which can be obtained by using the variational approximation and the Kantorowitch method [38,39]. 


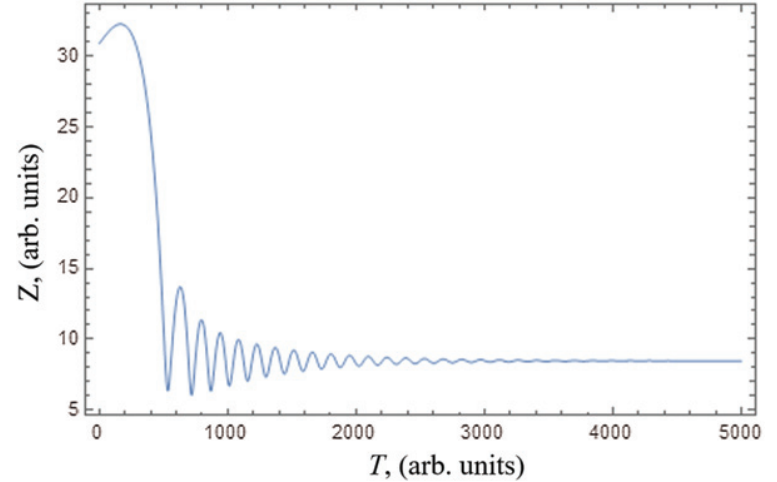

Fig. 1: Soliton temporal width evolution obtained by direct numerical simulation in the framework of variational approximation (red point in fig. 2): $\tau=0.1, \Lambda=-0.01, \kappa=0.009$, $\sigma=0.002$. The initial amplitude is of $\psi_{0}(z=0)=0.1$, $\theta(z=0)=0, \zeta(z=0)=0$, and the initial temporal and spatial widths correspond to the non-dissipative case considered in [32].

The $T$-independent solutions for the soliton parameters are

$$
\begin{aligned}
& \zeta=\frac{8 \sqrt{15} \tau}{\pi^{2} \mathrm{Z}^{2}\left(\sqrt{15+128 \tau^{2}}+\sqrt{15}\right)} \\
& \theta=-\frac{1}{4}\left(2 \kappa \mathrm{P}^{2}+\sigma \psi_{0}^{2} \mathrm{Z}\right) \\
& \psi_{0}^{2}=6\left(1-\mathrm{P}^{4}-\kappa^{2} \mathrm{P}^{8}\right) / \\
& \mathrm{P}^{2}\left(\sqrt{9 \sigma^{2}\left(1-\mathrm{P}^{4}\right) \mathrm{Z}^{2}+6 \kappa \sigma \mathrm{ZP}^{4}+1}+3 \kappa \sigma \mathrm{ZP}^{4}+1\right) .
\end{aligned}
$$

The solution for $\mathrm{Z}$ and the final equation for $\mathrm{P}^{2}$ are too cumbersome, and we do not explicitly show them here [39]. The equation for $\mathrm{P}^{2}$ contains radicals and has to be solved numerically. Direct numerical simulations in the framework of the variational approximation (figs. 1 and 2(b)) demonstrate that these solutions are stable within a broad range of initial conditions, for a confined range of the dissipative parameters $\Lambda, \tau$, and $\kappa$. Exploring this range should be closely connected with direct numerical simulations of eq. (1), and it will be presented elsewhere.

The dependence of soliton parameters on the various terms in eq. (1) is shown in figs. 2, 3. The specific values of our dimensionless parameters are close to those considered in [30]. Figure 2 illustrates the dependence of the soliton spatial size on graded dissipation $(\kappa)$, kinetic cooling $(\tau)$, and gain saturation $(\sigma)$, respectively. Gain saturation $\sigma$ leads to a decrease of the effective potential width $\chi$, which results in a spatial compression (or squeezing) of the soliton. This spatial squeezing is reduced as the graded potential $\kappa$ grows larger (see fig. 2). Correspondingly, the soliton amplitude $\psi_{0}^{2}$ decreases (see fig. 3). One can see from these figures that a tenfold decrease of the kinetic cooling parameter $\tau$ leads to a significant increase of the soliton spatial squeezing, accompanied by a substantial growth of its amplitude.
Necessary, but not sufficient, condition for 1Ddissipative soliton stability is the positiveness of $\Lambda_{s}$ (i.e., the negativity of net gain), which means suppressing the background excitation. An additional nonlinear gain term is required for supporting a soliton (e.g., see $[28,29,40])$. In our case, we do not assume such a mechanism, and a soliton with higher intensity $\left|\psi_{0}\right|^{2}$ is stabilized by its better confinement inside the graded dissipative potential. Obviously, the necessary but not sufficient condition for supporting such a soliton is the negativity of $\Lambda_{s}$ (i.e., the positiveness of the net gain inside the graded dissipative potential), which, nevertheless, does not guarantee against background excitation and multimode instabilities. Figure 4 demonstrates the dependence of the saturated gain on the graded dissipation parameter, for different values of gain saturation. As one can see, a lower gain saturation supports a soliton in the broader diapason of $\kappa$ (note that the soliton energy naturally decreases with $\kappa)$. It is essential that a "kinetic cooling" (i.e., $\tau$-growth) enhances soliton stability.

Thus, we should point out that results based on (2), i.e., the ground-state or fundamental mode assumption, provide important insights regarding soliton characteristics, including its stability, but are not conclusive, because they ignore the contribution of higher-order states (modes). Such a contribution can only be grasped by full numerical simulations of the dissipative eq. (1).

Dissipative mode cleaning. - For a specific model in optics that is analogous to BEC, we considered the master equation associated with spatiotemporal pulse propagation in a multimode fiber laser, based on active and nonlinear GRIN silica fibers. The graded dissipation in the fiber laser was characterized in terms of the previously discussed effective aperture size $\chi$. The presence of anomalous GVD, corresponding to a $z$-kinetic component, can be achieved by means of an appropriate waveguide compensation of the normal dispersion of silica when considering an active fiber dopant with gain centered around the carrier wavelength of $1.03 \mu \mathrm{m}$ (e.g., ytterbium). For example, this can be obtained by the microstructuring of a fiber [41]. Alternatively, the carrier wavelength can be shifted deeper into the infrared region, e.g., using an erbium-doped fiber at $1550 \mathrm{~nm}$, in order to obtain anomalous material GVD. Following the analogy between BEC and photonics models, the longitudinal coordinate of the former corresponds to the local time coordinate $t$ of the latter, whereas the GVD value defines the normalization of the $z$-coordinate for BEC. Let us recall that the time coordinate $T$ for BEC corresponds to the propagation distance along the optical fiber. We used the finite-element methods for the numerical simulations of eq. (1), under the condition of saturated gain, i.e., with $\Lambda_{s} \ll 1$.

In the absence of dissipation, a beam in a GRIN fiber which is spatially confined by the external potential (see eq. (1)) exhibits a decay into a multitude of spatial patterns with complex dynamics: this corresponds to the 

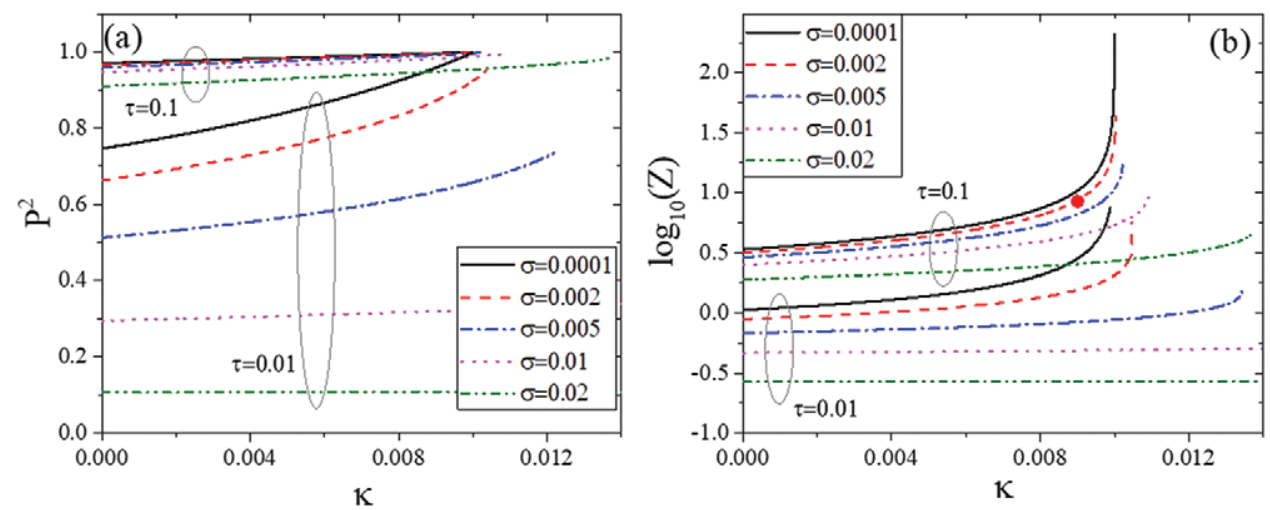

Fig. 2: (a) Transverse area of the soliton $\left(\mathrm{P}^{2}\right)$, and (b) its temporal width $\mathrm{Z}$ as a function of the gain saturation parameter $\sigma$, the graded dissipation parameter $\kappa$ and the kinetic cooling parameter (spectral dissipation in photonics) $\tau, \Lambda=-0.01$. The red point corresponds to the numerical result of fig. 1 .
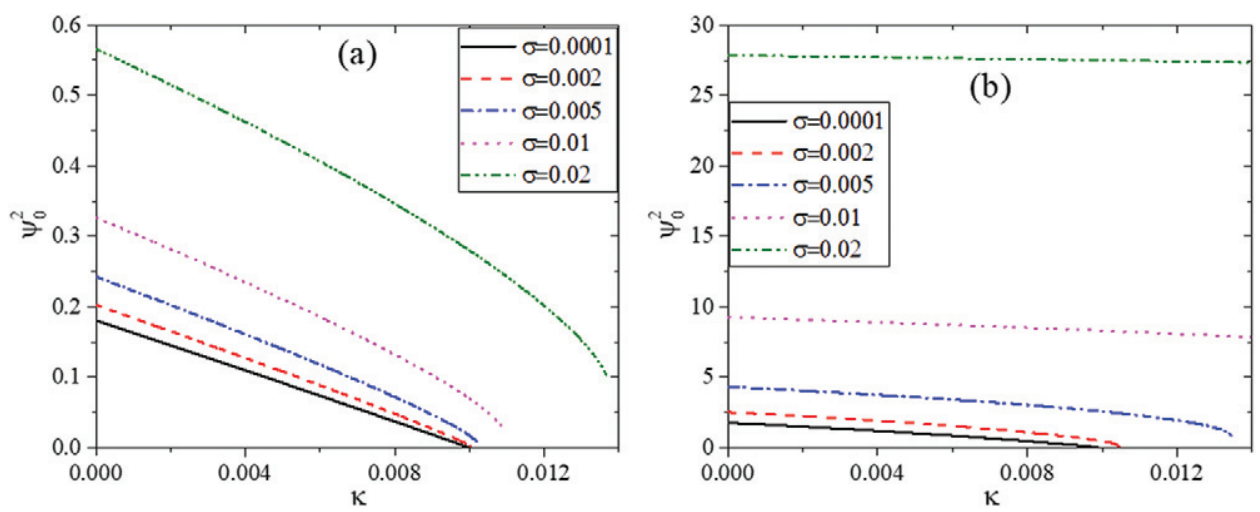

Fig. 3: Squared soliton amplitude for (a) $\tau=0.1$, and (b) $\tau=0.01$ as a function of the graded dissipation parameter $\kappa$, and the gain saturation parameter $\sigma, \Lambda=-0.01$.

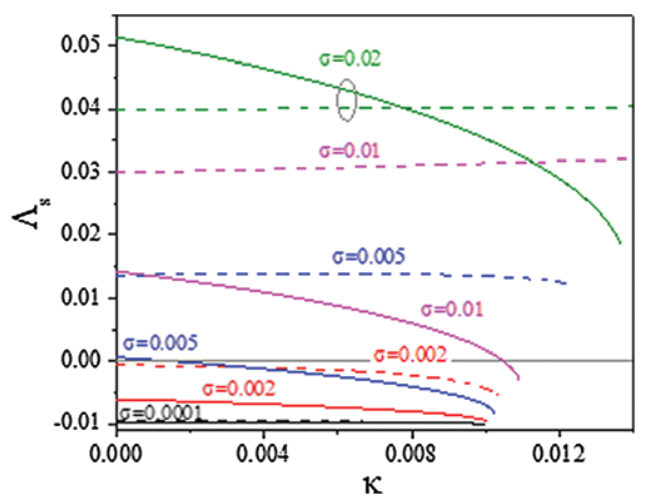

Fig. 4: Dependence of saturated net gain $\Lambda_{s}$ for the soliton solution (3) on a graded dissipation parameter $\kappa$ for different values of gain saturation parameter $\sigma$, and spectral dissipation ("kinetic cooling") $\tau=0.1$ (solid lines), 0.01 (dashed lines). $\Lambda=-0.01$.

presence of multimode beating, or self-imaging [34], (three spot-shoots are demonstrated in fig. 5).

The adjustment of graded dissipation provides mode cleaning [34]: as shown by fig. 6 , this is already effective for relatively short propagation distances, and moderate levels of $|\psi|^{2}$. This corresponds to the formation of a spatiotemporal soliton in the GRIN fiber [42]. As was shown, energy concentration in the fundamental mode can be interpreted in terms of two-dimensional hydrodynamic turbulence, and it occurs even in the case of random mode coupling $[43,44]$. Here, we note that mode cleaning and stable spatiotemporal soliton formation can be essentially enhanced by graded dissipation [33]. That directs to the mechanism of stable 2D-BEC formation by means of a manageable weak-dissipation. Nevertheless, dissipation provokes additional destabilizing effects. The first one involves soliton decay associated with a $\chi$-decrease (see table 1). A more interesting effect is the observation of stable spatiotemporal fragmentation, corresponding to multiple pulse train generation in a fiber laser (fig. 7(a)). This effect results from the decrease of graded dissipation (i.e., the $\chi$-parameter grows), so that the onset of dissipative mode cleaning is hampered. As a result, there is a decrease in the efficiency of field discrimination owing to the intensity dependence of the effective aperture or selffocusing, similar to the so-called Kerr lens mode-locking regime in a bulk laser [45]. In other words, the effective aperture becomes more "penetrable" for low-intensity fields. 


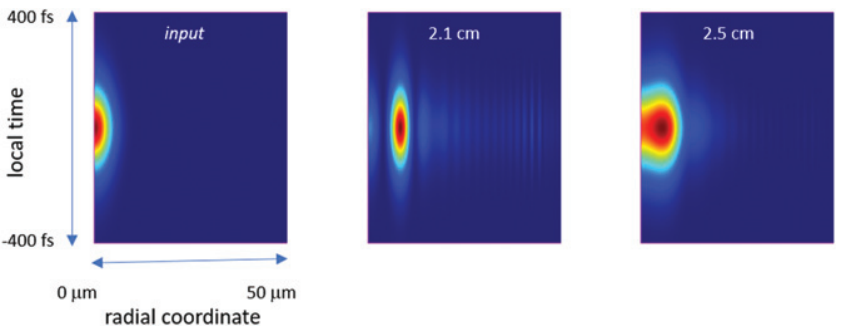

Fig. 5: Contour plots of $|\psi|^{2}$ at three propagation distances in the absence of dissipation $(\Lambda=0, \kappa=0)$. The following parameters characterize an MMF: the central wavelength is of $1.03 \mu \mathrm{m}$, the group-delay dispersion coefficient is of $-0.022 \mathrm{fs}^{2} / \mathrm{cm}$, the fiber core diameter is of $50 \mu \mathrm{m}$, and the confinement potential defined by the core/cladding refractive index difference is of 0.0103 (the cladding refractive index is of 1.457). The initial pulse with $\mathrm{P}=7 \mu \mathrm{m}$, corresponding to $10 \mu \mathrm{m}$ beam radius at $1 / e$-level has a temporal width $\mathrm{Z}=150 \mathrm{fs}$. The input peak power is of $100 \mathrm{~kW}$, and the nonlinear refractive index coefficient, defining the power normalization, is of $2.7 * 10^{-8} \mu \mathrm{m}^{2} / \mathrm{W}$. Local time and propagation distance in photonics correspond to unconfined coordinate $z$ and time $T$ for BEC in eq. (1). The dimensionless parameters could be obtained from [32].
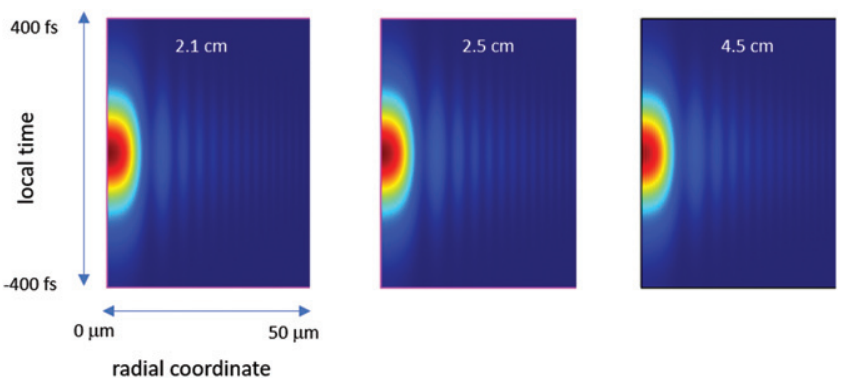

Fig. 6: Contour plots of $|\psi|^{2}$ for $\Lambda_{s}=-0.001, \chi=37 \mu \mathrm{m}$. $\tau$ is $1 / 100$ th of the GVD absolute value. Other parameters correspond to fig. 5 .

Table 1: Mode cleaning regimes for different dissipative parameters (a propagation length is mentioned, the maximum propagation length is of $5 \mathrm{~cm}$ ). $\tau$ is related to the anomalous dispersion value, $\Lambda_{s}=-0.001$. Other parameters correspond to fig. 5 .

\begin{tabular}{|ccc|}
\hline$\tau$ & $\chi, \mu \mathrm{m}$ & Regime \\
\hline 0.01 & 50 & Turbulence \\
0.01 & 40 & Multipulsing \\
0.01 & 37 & Regular at $5 \mathrm{~cm}($ fig. 6$)$ \\
0.01 & 35 & Spatial instability after $\approx 2.5 \mathrm{~cm}$ \\
0.01 & 32 & Decaying after $\approx 2.5 \mathrm{~cm}$ \\
\hline 0.05 & 50 & Turbulence \\
0.05 & 40 & Multipulsing \\
0.05 & 37 & Regular at $5 \mathrm{~cm}$ \\
0.05 & 35 & Spatial instability after $\approx 3.5 \mathrm{~cm}$ \\
0.05 & 32 & Decaying after $\approx 2.3 \mathrm{~cm}$ \\
\hline
\end{tabular}

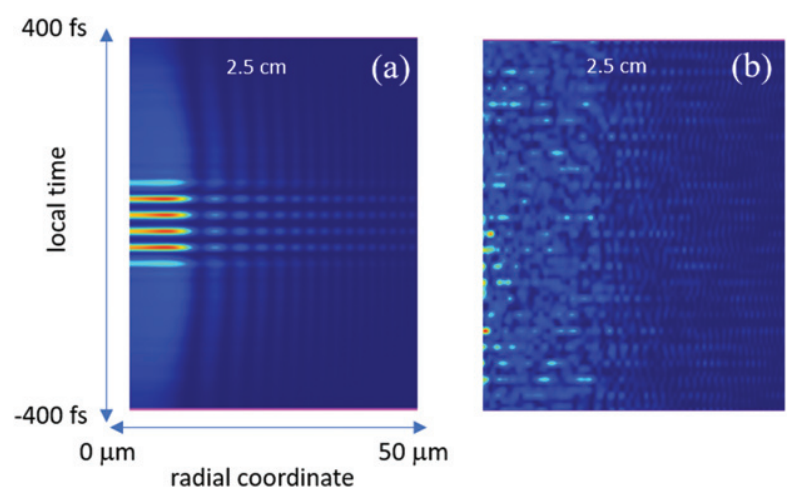

Fig. 7: Contour plots of $|\psi|^{2}$ for: (a) $\chi=40 \mu \mathrm{m}$, and (b) $\chi=$ $50 \mu \mathrm{m}, \tau=1 / 50$ th of the GVD absolute value; $\Lambda_{s}=-0.001$. Other parameters correspond to fig. 5 .

Table 2: Mode cleaning regimes for different dissipative parameters (a propagation length is mentioned, the maximum propagation length is of $5 \mathrm{~cm})$. $\tau$ related to the anomalous dispersion value is of $0.05, \Lambda_{s}=-0.01$. Other parameters correspond to fig. 5 .

\begin{tabular}{|cc|}
\hline$\chi, \mu \mathrm{m}$ & Regime \\
\hline 20 & Turbulence \\
10 & Temporal comb till $\approx 1.2 \mathrm{~cm}$ \\
5 & Temporal comb at $5 \mathrm{~cm}($ fig. 8$)$ \\
2 & Temporal comb till $\approx 1 \mathrm{~cm}$ \\
\hline
\end{tabular}

As was previously found $[26,32,46-49]$, the excitation of higher-order spatial modes may enhance multipulsing. In our case, the excitation of these modes results from reducing multimode cleaning, due to the growth of the effective aperture size. Thus, energy leaks into higherorder spatial modes, which diminishes effectiveness of discrimination between higher- and lower-intensity fields, and leads to multipulsing (see above). As a result, the beam tends to relax to a state which is characterized by multipulse generation (fig. 7(a)). This is akin to dissipative soliton multipulsing, which occurs as a result of the decrease of self-amplitude modulation (i.e., $\chi$-increase in our case) [7]. Such a transition to multipulsing can be interpreted as an excitation of the dissipative solitons' "internal modes" [50]. A further weakening of graded dissipation results in spatiotemporal turbulence, i.e., the generation of chaotically evolving disordered spatiotemporal structures, which occurs whenever the coherence between the mode patterns is fully broken (fig. 7(b)).

To summarize, there is a restricted range of parameters which provides effective mode cleaning by means of graded dissipation over extremely short propagation distances (tables 1, 2). In particular, a larger saturated gain $\left|\Lambda_{s}\right|$ may result in the generation of a temporal comb of solitons (fig. 8). 


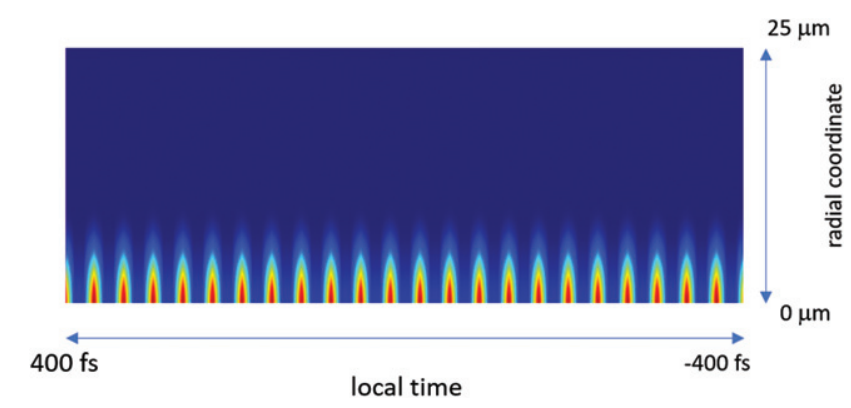

Fig. 8: Contour plot of $|\psi|^{2}$ for $\chi=5 \mu \mathrm{m}, \tau=1 / 20$ th of the GVD absolute value, $\Lambda_{s}=-0.01$. The propagation distance is of $5 \mathrm{~cm}$, other parameters correspond to fig. 5 .

Conclusion. - In this work, we analyzed the stabilization of $2 \mathrm{D}$ patterns (solitons) in a weakly dissipative BEC with a cigar-shaped confinement potential. The metaphorical modeling was based on the close analogy between nonlinear processes in 2D BEC and spatiotemporal soliton dynamics in a GRIN MMF. The underlying mathematical master equation is the generalized dissipative 2D+1 GPE, which was solved by the analytical but approximate variational approach, and by numerical methods. It was found that dissipative factors, such as 2D-graded dissipation, saturable gain, and kinetic cooling (spectral dissipation), play a crucial role in stabilizing spatiotemporal solitons. In photonics, one can treat such a stabilization as a manifestation of mode cleaning in an MMF, which is enhanced by dissipation and can be realized on extremely short propagation distances. That could provide a spatiotemporal mode-locking mechanism in a fiber laser, akin to the regime of distributed Kerr lens mode locking in solid-state lasers.

We identified the main scenarios leading to the destabilization of coherent spatiotemporal nonlinear wave structures. The prevailing of graded dissipation, i.e., a too narrow effective aperture, causes $2 \mathrm{D}$ soliton dissolution. The growth of the aperture size results in the formation of stable 2D soliton. A further aperture widening causes a soliton splitting initially, i.e., the formation of 2D patterns, or spatiotemporal multipulse generation in photonics. When the contribution of graded dissipation becomes too weak, the condensate loses its coherence, and spatiotemporal turbulence develops. The main practical significance of the obtained results lies in the possibility of mass/energy scaling of coherent 2D matter wave structures, and the demonstration of stable spatiotemporal mode locking in MMF lasers.

$$
* * *
$$

This work has received funding from the European Union Horizon 2020 research and innovation program under the European Research Council Advanced Grant No. 740355 (STEMS), the Marie Skłodowska-Curie Grant
No. 713694 (MULTIPLY), and the Russian Ministry of Science and Education Grant No. 14.Y26.31.0017.

\section{REFERENCES}

[1] Kevrekidis P. G., Frantzeskakis D. J. and Carretero-González R. (Editors), Emergent Nonlinear Phenomena in Bose-Einstein Condensates (SpringerVerlag) 2008, 405.

[2] Dalfono F., Giorgini S., Pitaevskit L. P. and Stringari S., Rev. Mod. Phys., 71 (1999) 463.

[3] Aschieri P., Garnier J., Michel C., Doya V. and Picozzi A., Phys. Rev. A, 83 (2011) 033838.

[4] Klaers J., Schmitt J., Vewinger F. and Weitz M., Nature, 468 (2010) 545.

[5] Kasprzak J., Richard M., Kundermann S., BaAs A., Jeambrun P., Keeling J. M. J., Marchetti F. M., Szymańska M. H., André R., Staehli J. L., Savona V., Littlewood P. B., Deveaud B. and Dang Le Si, Nature, 443 (2006) 409.

[6] Garnier J., Lisak M. and Picozzi A., J. Opt. Soc. Am. B, 29 (2012) 2229.

[7] Skiadas Ch. H. and Skiadas Ch. (Editors), Handbook of Applications of Chaos Theory (CRC Press) 2016, 928.

[8] Zakharov V. E. and Nazarenko S. V., Physica D, 201 (2005) 203.

[9] Aknermans E. and Montambaux G., Mesoscopic Physics of Electrons and Photons (Cambridge University Press) 2011, 608.

[10] Abdullaev F. Kh., Gammal A., Kamchatnov A. M. and Tomio L., Int. J. Mod. Phys. B, 19 (2005) 3415.

[11] Dauxois T. and Peyrard M., Physics of Solitons (Cambridge University Press) 2006, 422.

[12] Stegeman G. I. and Segev M., Science, 286 (1999) 1518.

[13] Kelley P. L., Phys. Rev. Lett., 15 (1965) 1005.

[14] Bradley C. C., Sackett C. A. and Hulet R. G., Phys. Rev. Lett., 78 (1997) 985.

[15] Malomed B. A., Eur. Phys. J. ST, 225 (2016) 2507.

[16] Mihalache D., Mazilu D., Malomed B. A. and LedERER F., Phys. Rev. A, 73 (2006) 043615.

[17] Shtyrina O. V., Fedoruk M. P., Kivshar Y. S. and Turitsyn S. K., Phys. Rev. A, 97 (2018) 013841.

[18] Khaykovich L., Schreck F., Ferrari G., Bourdel T., Cubizolles J., Carr L. D., Castin Y. and SaLOMON C., Science, 2961290.

[19] Abdullaev F. Kh., Kamchatnov A. M., Konotop V. V. and Brazhnyi V. A., Phys. Rev. Lett., 90 (2003) 230402.

[20] Saito H. and Ueda M., Phys. Rev. Lett., 90 (2003) 040403.

[21] Montesinosa G. D., Pérez-García V. M. and Torres P. J., Physica D, 191 (2004) 193.

[22] Adhikari S. K., Phys. Rev. A, 69 (2004) 063613.

[23] Malomed B. A., Soliton Management in Periodic Systems (Springer) 2006, p. 180.

[24] Agrawal G. E., Applications of Nonlinear Fiber Optics (Academic Press) 2001, p. 472.

[25] Trillo S. and Torruellas W. (Editors), Spatial Solitons (Springer-Verlag) 2001, p. 454.

[26] Kartashov Y. V., Astrakharchik G. E., Malomed B. A. and Torner L., Nat. Rev. Phys., 1 (2019) 185. 
[27] Editorial, Nat. Photon., 8 (2014) 1.

[28] Abdullaev F. Kh., Gammal A., Da Luz H. L. F. and Tomio L., Phys. Rev. A, 76 (2007) 043611.

[29] Bludov Yu. V., Konotop V. V. and Akhmediev N., Phys. Rev. A, 80 (2009) 033610.

[30] Bludov Yu. V. and Konotop V. V., Phys. Rev. A, 81 (2010) 013625.

[31] Akhmediev N. and Ankiewicz A. (Editors), Dissipative Solitons (Springer-Verlag) 2005, 448.

[32] Kalashnikov V. L. and Wabnitz S., Phys. Rev. A, 102 (2020) 023508.

[33] Wright L. W., Christodoulides D. N. and Wise F. W., Science, 358 (2017) 94.

[34] Krupa K., Tonello A., Barthélémy A., Mansuryan T., Couderc V., Millot G., Grelu Ph., Modotto D., Babin S. A. and Wabnitz S., APL Photon., 4 (2019) 110901.

[35] Kolner B. H., IEEE J. Quantum Electron., QE-30 (1994) 1951.

[36] Salem R., Foster M. A. and Gaeta A. L., Adv. Opt. Photon., 5 (2013) 274.

[37] Fedichev P. O., Kagan Yu., Shlyapnikov G. V. and Walraven J. T. M., Phys. Rev. Lett., 77 (1996) 2913.

[38] Chávez Cerda S., Cavalcanti S. and Hickmann J., Eur. Phys. J. D, 1 (1998) 313.

[39] Kalashnikov V. L., Variational approach to a fiberlaser spatial-temporal dissipative soliton: impact of gain saturation, Wolfram Mathematica notebook, DOI: 10.13140/RG.2.2.14399.33446.
[40] Harooni M. (Editor), High Power Laser Systems (IntechOpen) 2018, 212, p. 173.

[41] Zolla F., Renversez G., Nicolet A., Kuhlmey B., Guenneau S. and FelbacQ D., Foundations of Photonic Crystal Fibres (World Scientific Publishing) 2005, 376.

[42] Renninger W. H. and Wise F. W., Nat. Commun., 4 (2013) 719.

[43] Podivilov E. V., Kharenko D. S., Gonta V. A., Krupa K., Sidelnikov O. S., Turitsyn S., Fedoruk M. P., Babin S. A. and Wabnitz, Phys. Rev. Lett., 122 (2019) 103902.

[44] Fusaro A., Garnier J., Krupa K., Millot G. and Picozzi A., Phys. Rev. Lett., 122 (2019) 123902.

[45] Harooni M. (Editor), High Power Laser Systems (IntechOpen) 2018, 212, p. 181.

[46] Wright L. G., Sidorenko P., Pourbeyram H., Ziegler Z. M., Isichenko A., Malomed B. A., Menyuk C. R., Christodoulides D. N. and Wise F. W., Nat. Phys., 16 (2020) 565.

[47] Wright L. G., Renninger W. H., Christodoulides D. N. and Wise F. W., Opt. Express, 23 (2015) 3492.

[48] Ding E., Lefrancois S., Kutz J. N. and Wise F. W., IEEE J. Quantum Electron., QE-47 (2011) 597.

[49] Longhi S. and Janner D., J. Opt. B: Quantum Semiclass. Opt., 6 (2004) S303.

[50] Kalashnikov V. L. and Chernykh A., Phys. Rev. A, 75 (2007) 033820. 